\title{
Duplicação ureteral completa bilateral em mulher com Infecção do Trato Urinário (ITU) de repetição: relato de caso
}

\author{
Bilateral complete ureteral duplication in a woman with recurrent Urinary Tract Infection (UTI):
} case report

Duplicación ureteral completa bilateral en una mujer con Infección Urinaria recurrente (ITU): reporte de caso

Bárbara Queiroz de Figueiredo ORCID: https://orcid.org/0000-0003-1630-4597 Centro Universitário de Patos de Minas, Brasil

E-mail: barbarafigueiredo@unipam.edu.br

Ana Caroline Barcelos Souza ORCID: https://orcid.org/0000-0003-2616-4874 Universidade de Uberaba, Brasil E-mail: anacarolinebarcelossouza@gmail.com Bárbara Oliveira Vasconcelos Souto ORCID: https://orcid.org/0000-0003-3399-469X Centro Universitário de Patos de Minas, Brasil E-mail: barbarasouto710@hotmail.com Gardênia Silva Amorim ORCID: https://orcid.org/0000-0001-7888-8562 Centro Universitário de Atenas, Brasil E-mail: gardenia.sa@hotmail.com Pedro Dias Duarte

ORCID: https://orcid.org/0000-0001-6188-6608 Centro Universitário de Atenas, Brasil E-mail: pedrodias@unipam.edu.br

Rúbia Carla Oliveira

ORCID: https://orcid.org/0000-0002-7162-3497 Centro Universitário de Patos de Minas, Brasil E-mail: rubiacoliveira@unipam.edu.br

\begin{abstract}
Resumo
Introdução: os ureteres são estruturas tubulares finas bilaterais ( 3 a $4 \mathrm{~mm}$ ) que conectam os rins à bexiga urinária, transportando a urina da pelve renal para a bexiga. As camadas musculares são responsáveis pela atividade peristáltica que o ureter usa para mover a urina dos rins para a bexiga. Objetivo: expor um caso clínico de paciente com duplicidade ureteral completa bilateral, associado a episódios frequentes de infecção do trato urinário. Metodologia: trata-se de um estudo de caso clínico com perspectiva qualitativa e descritiva, que consiste em uma pesquisa em que, em geral, ocorre com coleta direta de dados, cujo o pesquisador é o instrumento indispensável. Relato de caso: trata-se de paciente do sexo feminino, 18 anos de idade, com episódios frequentes, desde os 14 anos, de infecções do trato urinário e queixas de sensação de esvaziamento vesical incompleto, em tratamento com o urologista. Refere-se que após as infecções, intensificam-se a presença de corrimento vaginal constante, em grande quantidade, com odor forte, no entanto, sem ardência. Aos 15 anos, obteve diagnóstico de duplicação ureteral completa bilateral, por meio de tomografia computadorizada de abdome total e com posterior administração venosa de contraste. Conclusão: Muitas das alterações morfológicas ureterais podem ser avaliadas por meio da tomografia computadorizada, devido seus mais modernos avanços tecnológicos tem contribuído nos últimos anos para uma melhor caracterização das alterações morfológicas, sendo fundamental no diagnóstico das anomalias congênitas, orientando melhor decisões terapêuticas clínicas e cirúrgicas e atuando como ferramenta essencial na identificação de complicações associadas.
\end{abstract}

Palavras-chave: Ureteres; Anomalias; Trato urinário; ITU.

\begin{abstract}
Introduction: The ureters are bilateral thin tubular structures ( 3 to $4 \mathrm{~mm}$ ) that connect the kidneys to the urinary bladder, carrying urine from the renal pelvis to the bladder. The muscle layers are responsible for the peristaltic activity that the ureter uses to move urine from the kidneys to the bladder. Objective: to present a clinical case of a
\end{abstract}


patient with complete bilateral ureteral duplicity, associated with frequent episodes of urinary tract infection. Methodology: this is a clinical case study with a qualitative and descriptive perspective, which consists of a research that, in general, takes place with direct data collection, in which the researcher is the indispensable instrument. Case report: this is a female patient, 18 years old, with frequent episodes, since the age of 14, of urinary tract infections and complaints of feeling of incomplete bladder emptying, under treatment with a urologist. It is reported that after infections, the presence of constant vaginal discharge is intensified, in large quantities, with a strong odor, however, without burning. At 15 years of age, he was diagnosed with complete bilateral ureteral duplication, by means of computed tomography of the total abdomen, with subsequent intravenous administration of contrast. Conclusion: Many of the ureteral morphological changes can be evaluated using computed tomography, due to its most modern technological advances, it has contributed in recent years to a better characterization of morphological changes, being essential in the diagnosis of congenital anomalies, better guiding clinical and surgical therapeutic decisions and acting as an essential tool in identifying associated complications.

Keywords: Ureters; Anomalies; Urinary tract; ITU.

\section{Resumen}

Introducción: Los uréteres son estructuras tubulares delgadas bilaterales ( 3 a $4 \mathrm{~mm}$ ) que conectan los riñones con la vejiga urinaria, transportando la orina desde la pelvis renal a la vejiga. Las capas musculares son responsables de la actividad peristáltica que utiliza el uréter para mover la orina desde los riñones a la vejiga. Objetivo: presentar un caso clínico de un paciente con duplicidad ureteral bilateral completa, asociada a frecuentes episodios de infección del tracto urinario. Metodología: se trata de un estudio de caso clínico con perspectiva cualitativa y descriptiva, que consiste en una investigación que, en general, se realiza con recolección directa de datos, en la que el investigador es el instrumento indispensable. Caso clínico: se trata de una paciente de 18 años, con frecuentes episodios, desde los 14 años, de infecciones del tracto urinario y quejas de sensación de vaciamiento incompleto de la vejiga, en tratamiento con urólogo. Se informa que después de las infecciones se intensifica la presencia de flujo vaginal constante, en grandes cantidades, con un olor fuerte, sin embargo, sin quemar. A los 15 años se le diagnosticó duplicación ureteral completa bilateral, mediante tomografía computarizada de abdomen total, con posterior administración de contraste intravenoso. Conclusión: Muchos de los cambios morfológicos ureterales pueden ser evaluados mediante tomografía computarizada, debido a sus más modernos avances tecnológicos, ha contribuido en los últimos años a una mejor caracterización de los cambios morfológicos, siendo fundamental en el diagnóstico de anomalías congénitas, mejor orientando clínica y decisiones terapéuticas quirúrgicas y actuando como herramienta fundamental en la identificación de las complicaciones asociadas.

Palabras clave: Uréteres; Anomalías; Tracto urinario; ITU.

\section{Introdução}

Os ureteres são estruturas tubulares finas bilaterais (3 a $4 \mathrm{~mm}$ ) que conectam os rins à bexiga urinária, transportando a urina da pelve renal para a bexiga. As camadas musculares são responsáveis pela atividade peristáltica que o ureter usa para mover a urina dos rins para a bexiga. Embriologicamente, o ureter origina-se do botão ureteral, que é uma protrusão do ducto mesonéfrico, parte do desenvolvimento do sistema geniturinário (Lascay et al. 2021).

Os ureteres começam na junção ureteropélvica (JUP) dos rins, que se encontram posteriormente à veia e artéria renais no hilo. Os ureteres então viajam inferiormente dentro da cavidade abdominal. Eles passam sobre (anteriormente) o músculo psoas e entram na bexiga na face posterior da bexiga no trígono. Três áreas ao longo do trajeto do ureter são clinicamente significativas para o alojamento de cálculos renais. Essas áreas são: a junção ureteropélvica (UPJ), a junção ureterovesical (UVJ) e o cruzamento das artérias ilíacas comuns. O UPJ é onde a pelve do rim faz a transição para o ureter e o UVJ é onde os ureteres entram na bexiga (Soriano et al. 2021).

O sistema coletor renal é sede frequente de variações anatômicas com respeito a tamanho, forma, grau de ramificação e grau de rotação em relação ao hilo renal. A estenose da junção ureteropélvica (JUP) é a anomalia urinária mais comum na infância e mais frequente nas crianças do sexo masculino, normalmente sendo diagnosticada no primeiro ano de vida, mas que pode seguir não diagnosticada até a vida adulta, sendo nesta faixa etária mais comum em mulheres (Brant et al. 2007). Nesta afecção, há um estreitamento da JUP, geralmente à esquerda, que pode ser decorrente de lesão intrínseca muscular ou de descontinuidade funcional nesse segmento, que impede o esvaziamento adequado da pelve renal resultando em hidronefrose (Kim, 2012). 
Porém, a maior parte das anomalias da pelve renal e do ureter apresenta-se como duplicidade do sistema coletor, causa comum de assimetria de dimensões entre os rins durante a infância e que ocorre em $1 \%$ a $2 \%$ da população, sendo mais comum no sexo feminino. Esta duplicação pode ser completa ou incompleta, com a forma unilateral mais comum que a bilateral, e frequentemente está associada a várias complicações. O rim com duplo sistema coletor tem maior tamanho, sobretudo em seu eixo longitudinal, e maior volume do parênquima (Gun et al. 2012).

$\mathrm{Na}$ duplicidade completa há dois sistemas coletores para um único rim e dois ureteres de um mesmo lado, que desembocam em orifícios separados. De acordo com a regra de Weigert-Meyer, o ureter que drena a parte superior passa pela parede da bexiga urinária para se inserir inferior e medialmente ao local normal de sua inserção. Com frequência, sua inserção é defeituosa, associada a ureterocele, e quando ectópica, pode desembocar na uretra posterior, na vagina ou na vulva. O ureter do segmento inferior insere-se próximo ao local de normalidade e está sujeito a refluxo vesicoureteral devido à distorção que sofre ao atravessar a parede da bexiga urinária associada a ureterocele. À radiografia, a dilatação completa aparece como o sinal característico e bem conhecido, o dropping lily. Complicações da duplicação completa incluem infecções, refluxo vesicoureteral e obstrução da JUP. O refluxo no sistema coletor do segmento inferior pode produzir cicatrizes e deformidades deste segmento (Dyer et al. 2004).

As variações anatômicas do ureter e sua relação com as estruturas adjacentes são, portanto, importantes na perspectiva acadêmica e clínica para preservar as funções renais. A duplicação dos ureteres resulta da divisão precoce do botão ureteral em dois ou mais de forma completa ou incompleta (ureter bífido). (Sailaja et al. 2014). Sob esse contexto, a duplicidade do ureter pode ser encontrada em pacientes de maneira assintomática. Entretanto, pode ser causa de infecções recorrentes do trato urinário (ITU), incontinência urinária e urolitíase (Croitoru et al. 2007).

As complicações mais comuns na duplicidade completa são a ureterocele, que consiste na dilatação cística do ureter intravesical com obstrução, correspondente ao polo superior renal, com acúmulo de urina podendo obstruir também a saída da bexiga e pode ocasionar ureterohidronefrose e ITU; refluxo da urina da bexiga para o polo inferior do rim causando também infecção urinária; ectopia do ureter superior desembocando na uretra, ao lado do hímen ou na vagina na menina, e nos meninos na uretra posterior e até no ducto ejaculatório determinando orquite de repetição (Lascay et al. 2021).

O diagnóstico dos pacientes sintomáticos é feito na infância, sendo muito menos comum detectar a duplicidade nos pacientes adultos. Os exames de imagem que podem ser utilizados para diagnóstico são a ultrassonografia, cistouretrograma miccional (VCUG) e a tomografia computadorizada com o sistema MDCT (técnica de varredura rápida com avaliação 3D). O tratamento é cirúrgico nos pacientes sintomáticos, para solucionar a incontinência e possíveis ITUs, e, assim, prevenir outras complicações posteriores. Em suma, é uma anomalia que, nos casos complicados, o diagnóstico é usualmente feito, sem muitas dificuldades, com exames específicos logo ao nascer, proporcionando excelentes resultados terapêuticos quando necessitam de cirurgia. (Park et al. 2016). Sob essa perspectiva, o objetivo do presente estudo consiste em expor um caso clínico de paciente com duplicidade ureteral completa bilateral, associado a episódios frequentes de infecção do trato urinário.

\section{Metodologia}

Este artigo trata-se de um estudo de caso clínico com perspectiva qualitativa e descritiva, que consiste em uma pesquisa em que, em geral, ocorre com coleta direta de dados, cujo o pesquisador é o instrumento indispensável. O presente estudo contou com o aporte de trabalhos acadêmicos entre os anos de 2001 e 2020. Por meio do acesso às principais bases de dados, a saber: dados National Library of Medicine (PubMed MEDLINE), Scientific Electronic Library Online (Scielo), Cochrane Database of Systematic Reviews (CDSR), Google Scholar, Biblioteca Virtual em Saúde (BVS) e EBSCO Information Services, no período compreendido entre março e julho de 2021. 
O Termo de Consentimento Livre e Assistido (TCLE) foi assinado pelo paciente para coleta de dados neste estudo. Trata-se de um estudo de um relato de caso clínico, de abordagem qualitativa, com delineamento descritivo, de caráter narrativo e reflexivo, como intuito de proporcionar a disseminação de conhecimento na área em que se insere à comunidade científica e clínica. Os dados foram coletados em prontuário médico da USF Itamarati, associado aos exames de imagem realizados pela paciente.

\section{Relato de Caso}

Trata-se de paciente do sexo feminino, 18 anos de idade, com episódios frequentes, desde os 14 anos, de infecções do trato urinário e queixas de sensação de esvaziamento vesical incompleto, em tratamento com o urologista. Refere-se que após as infecções, intensificam-se a presença de corrimento vaginal constante, em grande quantidade, com odor forte, no entanto, sem ardência. Ademais, alega hábitos urinários regulares, no entanto, ciclos menstruais irregulares e em grande volume.

Aos 15 anos, obteve diagnóstico de duplicação ureteral completa bilateral, por meio de tomografia computadorizada de abdome total e com posterior administração venosa de contraste, que, por sua vez, foi eliminado satisfatoriamente por ambos os rins. O exame demonstrou mínima quantidade de líquido na pelve, achado habitualmente usual e destituído de significado clínico e demais órgãos pélvicos visibilizados sem alterações. A Imagem 1 mostra a duplicidade completa bilateral, com a junção dos ureteres ao nível da junção uterovesical, sem fator obstrutivo detectável ou retardo na eliminação do contraste endovenoso.

Imagem 1: Duplicidade do sistema coletor.

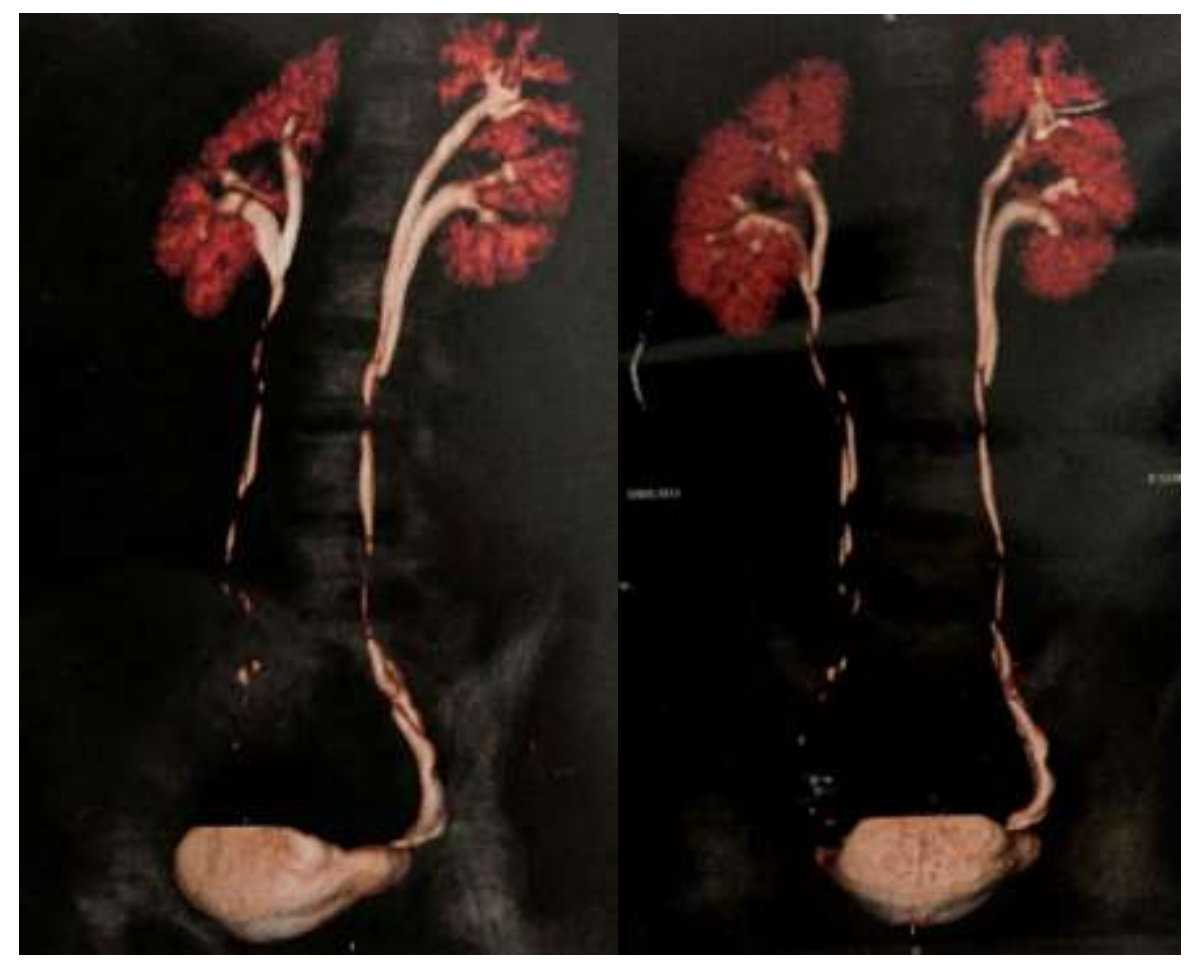

Fonte: Dados da pesquisa (2021).

Ao exame de ultrassonografia, constatou-se regiões ureterais sem alterações, dilatações discretas pielocalinais na metade inferior do rim direito e calicinal superior isolada ao rim esquerdo. Na duplicidade completa, conforme ilustrado pelo caso, há dois sistemas coletores para um único rim e dois ureteres de um mesmo lado, que desembocam em orifícios separados. 


\section{Discussão}

A duplicação ureteral pode ser completa ou incompleta e geralmente é acompanhada por várias complicações. A duplicação incompleta está mais frequentemente associada a refluxo ureteroureteral ou obstrução da junção ureteropélvica do polo inferior do rim (Hanson et al. 2007). A duplicação completa está mais frequentemente associada a refluxo vesicoureteral, ureterocele ectópica ou inserção ureteral ectópica, todos os quais são mais comuns em meninas do que em meninos. O refluxo vesicoureteral afeta o polo inferior e pode ser superado, como em sistemas não duplicados (El-Ghar et al. 2013). A ureterocele ectópica e a inserção ureteral ectópica afetam o polo superior. A ureterocele ectópica produz um defeito de enchimento de tamanho variável na bexiga; pode ser identificada com estudos de material de contraste ou ultrassom (Fernbach et al. 2007).

Uma quarta complicação, a obstrução da junção ureteropélvica, ocorre apenas no polo inferior e é vista em mais meninos do que meninas. Variantes anatômicas ou anomalias, bem como técnicas de imagem abaixo do ideal, podem simular ou ocultar a duplicação, tornando o diagnóstico difícil (Toprak et al. 2007). No entanto, a familiaridade com a embriologia da duplicação e uma consciência das armadilhas potenciais da urografia excretora e uretrocistografia miccional promoverão uma compreensão das aparências variadas e complicações associadas da duplicação incompleta e completa (Tanrıverdi et al. 2007). Variantes anatômicas ou anomalias, bem como técnicas de imagem abaixo do ideal, podem simular ou ocultar a duplicação, tornando o diagnóstico difícil. No entanto, a familiaridade com a embriologia da duplicação e uma consciência das armadilhas potenciais da urografia excretora e uretrocistografia miccional promoverão uma compreensão das aparências variadas e complicações associadas da duplicação incompleta e completa (Oge et al. 2001).

\section{Considerações Finais}

Muitas das alterações morfológicas ureterais podem ser avaliadas por meio da tomografia computadorizada, devido seus mais modernos avanços tecnológicos tem contribuído nos últimos anos para uma melhor caracterização das alterações morfológicas, sendo fundamental no diagnóstico das anomalias congênitas, orientando melhor decisões terapêuticas clínicas e cirúrgicas e atuando como ferramenta essencial na identificação de complicações associadas. Dessa forma, o presente estudo é capaz de ilustrar um caso de duplicação ureteral bilateral completa associado a episódios de infeções repetitivas no trato urinário, em paciente jovem do sexo feminino. Assim, representa um empirismo importante para a prática clínica, ao evidenciar uma anormalidade anatômica associada a uma importante patologia como as infecções do trato urinário, haja vista que como diagnóstico diferencial de incontinência urinária, as malformações do sistema urinário devem ser lembradas, para que se possa fazer o diagnóstico correto e precoce dessa patologia.

\section{Referências}

Brant, W. E., et al. (2007). Fundamentals of diagnostic radiology. (3a ed.), Lippincott Williams \& Wilkins.

Croitoru, S., et al. (2007). Duplicated ectopic ureter with vaginal insertion: 3d ct urography with iv and percutaneous contrast administration. AJR, 189 (1), $272-274$.

Dyer, R. B., et al. (2004). Classic signs in uroradiology. Radiographics, 24 (1), 247-280.

El-Ghar, M. A. \& El-Diasty, T. (2013). Inserção ectópica do ureter na vesícula seminal. World J Radiol., 5 (9): $349-51$.

Fernbach, S. K., Feinstein, K. A., Spencer, K. \& Lindstrom, C. A. (2007). Duplicação ureteral e suas complicações. Radiografias, 17 (3), $109-117$.

Gun, S., et al. (2012). Fusão renal completa em criança com infecção recorrente do trato urinário. Radiol Bras., 45 (1), $233-234$.

Hanson, G. R., Gatti, J. M., Gittes, G. K. \& Murphy, J. P. (2007). Diagnóstico de ureter ectópico como causa de incontinência urinária. J Paediatr Urol., 3 (1): $53-57$.

Kim, S. H. (2012). Radiology illustrated - uroradiology. (2a ed.), Springer-Verlag.

Lescay, H. A., et al. (2021). Anatomy, Abdomen and Pelvis, Ureter. StatPearls. 
Research, Society and Development, v. 10, n. 12, e90101220174, 2021

(CC BY 4.0) | ISSN 2525-3409 | DOI: http://dx.doi.org/10.33448/rsd-v10i12.20174

Oge, O., Ozeren, B. \& Sonmez, F. (2001). Cirurgia poupadora de néfrons em um sistema duplex associado a um ureter ectópico vaginal. Paediatr Nephrol.,16 (3), 1135-1136.

Park, A., et al. (2016). Compreendendo o ureter: desafios e oportunidades. J Endourol.,30 (1), 34-36.

Sailaja, T. K., et al. (2014). Uma rara anomalia do ureter e seu aspecto de desenvolvimento - Relato de caso. MRIMS J Health Sci, 2(1), $102-104$.

Soriano, R. M., et al. (2021). Anatomy, Abdomen and Pelvis, Kidneys. StatPearls.

Tanrıverdi, I., Ulman, I. \& Avanoglu, A. (2007). Endoscopik nefroureterectomia e experiência em nefroureterectomia de pólo superior usando intra ou retro peritoneal. J Associação Turca de Cirurgiões Pediátricos, 21 (1): 59-61.

Toprak, U., Erdogan, A., Pasaoglu, E. \& Karademir, M. A. (2007). A importância da tomografia computadorizada da fase da pielografia no diagnóstico do ureter vaginal ectópico. J Ankara University Faculty of Medicine, 60 (1), 35-37. 